\title{
Preparation of Folate-Conjugated Pluronic F127/Chitosan Core-Shell Nanoparticles Encapsulating Doxorubicin for Breast Cancer Treatment
}

\author{
Chawan Manaspon, ${ }^{1}$ Kwanchanok Viravaidya-Pasuwat, ${ }^{1,2}$ and Nuttaporn Pimpha ${ }^{3}$ \\ ${ }^{1}$ Biological Engineering Program, King Mongkut's University of Technology Thonburi, Bangkok 10140, Thailand \\ ${ }^{2}$ Department of Chemical Engineering, King Mongkut's University of Technology Thonburi, Bangkok 10140, Thailand \\ ${ }^{3}$ National Nanotechnology Center, Thailand Science Park, Paholyothin Road, Pathumthani 12120, Thailand \\ Correspondence should be addressed to Kwanchanok Viravaidya-Pasuwat, kwanchanok.vir@kmutt.ac.th \\ Received 6 August 2011; Revised 5 November 2011; Accepted 11 November 2011 \\ Academic Editor: Zhongkui Hong
}

Copyright (C) 2012 Chawan Manaspon et al. This is an open access article distributed under the Creative Commons Attribution License, which permits unrestricted use, distribution, and reproduction in any medium, provided the original work is properly cited.

A targeting drug delivery system using folate-conjugated pluronic F127/chitosan core-shell nanoparticles was developed to deliver doxorubicin (DOX) to the target cancer cells. First, DOX was encapsulated in pluronic F127 micelle cores in the presence of sodium dodecyl sulfate (SDS) by a self-assembly method. To form a shell, a layer of either chitosan or folate-conjugated chitosan was deposited onto the pluronic micelles. The encapsulation efficiency was approximately $58.1 \pm 4.7 \%$. The average size of the core-shell nanoparticles was $37.4 \pm 2.0 \mathrm{~nm}$, while the zeta potential was $12.9 \pm 2.3 \mathrm{mV}$, indicating the presence of a shell layer and more stable particles. In an in vitro DOX release study, an initial burst release, followed by a sustained release, was observed within 24 hours. In addition, the core-shell nanoparticles showed greater cytotoxicity towards MCF-7 cells than free DOX, suggesting a better therapeutic efficacy in treating cancer.

\section{Introduction}

Doxorubicin (DOX), a poorly soluble drug, has been one of the most commonly used chemotherapeutic agents to treat several types of cancer, including breast cancer, in the past decade. Although it exhibits high efficacy, DOX is also toxic towards healthy tissues, causing life-threatening side effects [1-3]. Therefore, a better DOX delivery strategy is needed. DOX-formulated nanoparticles are thought to improve the drug efficacy, while reducing side effects. Several delivery systems for DOX have been developed such as liposome [4, 5], micelles [6-8], hydrogel nanoparticles $[9,10]$, and DOXpolymeric conjugation [11-13]. Even though all of the above carriers were successfully synthesized in the laboratory, only DOX-encapsulated liposome, Doxil, is commercially available [4]. Unfortunately, Doxil has a short half-life in microcirculation and is unstable in blood stream, leading to side effects similar to that of free DOX [5]. Thus, the delivery system attempts have shifted towards more controlled and stable systems such as polymeric nanoparticles.
Polymeric nanoparticles are normally classified into two forms: hollow and core-shell nanoparticles [14]. For hollow nanoparticles, drugs are either encapsulated in their highly porous polymer matrix or conjugated with the polymer before the formation of nanoparticles. The most widely used polymers for hollow nanoparticles are PLGA and chitosan $[11,15,16]$. These nanoparticles, however, have low drug loading capacity, and their structures are somewhat unstable. Alternately, core-shell nanoparticles consist of two parts: the core and the shell, which are made from different polymers. Examples of core-shell nanoparticles are silica-coated chitosan nanoparticles [17], PMMA/chitosan core-shell nanoparticles [18], and chitosan coated poly(lactic-co-glycolic acid) (PLGA) [19]. Core-shell nanoparticles show greater thermal and chemical stability and lower cytotoxicity.

The core material of these nanoparticles can be chosen such that drug encapsulation efficiency is maximized. Recently, the interest in polymeric amphiphiles as core materials has been increasing, since this type of polymer can be used with both hydrophilic and hydrophobic compounds. 
One of the amphiphilic block copolymers widely studied in the drug delivery field is pluronic [20]. Pluronic is a triblock copolymer, composed of poly(ethylene oxide) (PEO) and poly(propylene oxide) (PPO) with a PEO-PPO-PEO structure. Pluronic spontaneously forms micelles with a diameter of approximately $30-50 \mathrm{~nm}$ at concentrations equal to or above the critical micelle concentration (CMC) [21-23]. Unfortunately, the use of pluronic micelles as prolonged drug carriers is not practical because of the particles' aggregation and structural changes when exposed to varying concentrations or temperatures. Several studies have shown that the particles' stability can be greatly improved after being coated with another polymeric layer with materials such as PLGA, polyvinylchloride (PVC), or chitosan [24]. Chitosan has been shown to be a favorable shell material due to its biocompatibility and biodegradability characteristics $[25,26]$. In addition, the cationic nature of chitosan enhances membrane adhesion, as well as membrane permeability, which are desirable for applications in drug delivery [16].

Another advantage of core-shell nanoparticles is that the polymeric shell can be conjugated with other molecules such as ligands or antibodies. These can bind to receptors on cell membrane with high affinity, allowing for targeting drug delivery. Labeling nanoparticles with ligands has been shown to give a higher cellular uptake via natural endocytosis pathways [27-30]. One of the most commonly used targeting ligand is folate or folic acid. Folateis known to be overexpressed on the surface of cancer cells, whereas it is expressed in low levels in normal tissue $[8,31,32]$.

In this study, we describe the preparation of pluronicchitosan core-shell nanoparticles conjugated with folate for DOX delivery. Briefly, pluronic and sodium dodecyl sulfate (SDS) self-assembled to form micelles, while encapsulating DOX within the core. Afterwards, an electrostatic interaction between the SDS and the chitosan led to the formation of chitosan shell layer on the micelles. The physical properties of the nanoparticles, including DOX encapsulation efficiency, average particle size, zeta potential, and in vitro drug release, were characterized. The cellular uptake and cytotoxicity studies of the nanoparticles against the breast cancer cell model, MCF-7 cell line, were also carried out to evaluate the targeting capability of the particles.

\section{Experimental}

2.1. Materials. Chitosan (medium molecular weight, $45 \mathrm{kDa}$; degree of deacetylation, $85 \%$ ) was purchased from Seafresh Chitosan Laboratory (Bangkok, Thailand). Pluronic F127 (Poloxamer 407) was donated by BASF Thailand. Folate, doxorubicin (DOX), N-(3-dimethylaminopropyl)-N-ethylcarbodiimide hydrochloride (EDC, protein seq. grade), and sodium acetate were purchased from Sigma (Missouri, USA). Ethanol (ACS grade) was purchased from Merck (Darmstadt, Germany). Lysozyme (Chicken egg white, ultrapure grade) and sodium dodecyl sulfate (SDS) were purchased from Bio Basic Inc. (Markham Ontario, Canada). Dulbecco's Modified Eagle Medium (DMEM), Trypsin-EDTA, Fetal Bovine Serum (FBS), and Vybant MTT cell proliferation kit were purchased from Invitrogen (Oregon, USA). Dimethyl sulfoxide (DMSO) was purchased from Amresco (OH, USA).

2.2. Preparation of Folate-Modified Chitosan. Folate conjugated chitosan was prepared according to the method described by Mansouri et al. [28] and Dubé et al. [33]. A concentrated solution of $2.40 \%(\mathrm{w} / \mathrm{v})$ of EDC and $2.20 \%(\mathrm{w} / \mathrm{v})$ of folate in DMSO was added to a $1 \%(\mathrm{w} / \mathrm{v})$ solution of chitosan in a $0.4 \mathrm{M}$ acetate buffer. The mixture was continuously stirred at room temperature in the dark for 16 hours. Afterwards, its pH was slowly adjusted to 9.0 and the solution was dialyzed against a phosphate buffer saline (PBS) with a pH of 7.5 for 2 days and against deionized water for 3 days. The folate-conjugated chitosan was isolated by lyophilization and its chemical structure confirmed with Fourier Transform Infrared Spectroscopy (FT-IR) before use.

2.3. Preparation of DOX-Loaded Core-Shell Nanoparticles. Appropriate amounts of pluronic and $25 \mu \mathrm{g} / \mathrm{mL}$ of DOX were first dissolved in a small volume of ethanol. The solution was left at room temperature to allow the solvent to evaporate completely, and the product was kept in a desiccator overnight [34]. The resulting powder was hydrated with deionized water at $60^{\circ} \mathrm{C}$ and sonicated for 20 minutes to yield DOX-loaded pluronic micelles. These micelles were subject to further modification by the addition of a $0.04 \%(\mathrm{w} / \mathrm{v})$ solution of SDS then incubated at room temperature for 1 hour to form closely packed micelles. The resulting solution was filtered through a $0.2 \mu \mathrm{m}$ pore size membrane filter (Minisart, Sartorius Stedium Biotech, Germany).

The pluronic micelle solution (the core) was gently mixed using a tube inversion method with a $0.005 \%(\mathrm{w} / \mathrm{v})$ solution of either chitosan or folate-conjugated chitosan in a $0.4 \mathrm{M}$ acetate buffer with $\mathrm{pH} 4.7$ for 20 minutes (suspension: chitosan solution ratio $=1: 1)[17,35]$. The sample was dialyzed for 3 days with a dialysis membrane (MWCO $7000 \mathrm{Da})$ against deionized water to remove excess SDS, DOX, and acetate buffer. Finally, the solutions were frozen and lyophilized for 8 hours.

2.4. Measurement of Drug Encapsulation Efficiency. The encapsulation efficiencies of DOX were calculated from the disappearance of DOX from its original solution. Free doxorubicin was measured using a fluorescence spectrophotometer $\left(\lambda_{\text {excitation }}=485 \mathrm{~nm}\right.$ and $\left.\lambda_{\text {emission }}=590 \mathrm{~nm}\right)$. The measurement was performed in triplicate. The percentage of doxorubicin encapsulation was determined using (1):

$$
\begin{aligned}
& \text { Encapsulation Efficiency (\%) } \\
& \begin{aligned}
= & \frac{\text { Amount of DOX inmicelles }}{\text { Amount of DOX initially added to the formation }} \\
& \times 100 \text {. }
\end{aligned}
\end{aligned}
$$

2.5. Particle Characterization. A transmission electron microscope (TEM; JEM 1220, Japan, $100 \mathrm{kV}$ voltage) was used to examine the morphology of the nanoparticles prepared 
in this study. The particle size distribution was determined using a photon correlation spectroscope (PCS, NanoZS 4700, Malvern Instruments, UK). The overall surface charges of the nanoparticles were measured using a zetasizer (NanoZs 4700, Mavern Instruments, UK). All measurements were repeated at least three times.

2.6. In Vitro Doxorubicin Release from Core-Shell Nanoparticles. Phosphate buffer saline (PBS) with $\mathrm{pH} 7.5$ containing $0.1 \%(\mathrm{w} / \mathrm{v})$ of lysozyme was used as a receiving solution in the study of in vitro DOX release from both chitosan and folate-conjugated chitosan nanoparticles [36, 37]. One milliliter of the $1.2 \%(\mathrm{w} / \mathrm{v})$ solution of nanoparticles was placed in a test tube and kept at $37^{\circ} \mathrm{C}$ without shaking. At a predetermined time, $0.1 \mathrm{~mL}$ of the sample was withdrawn and centrifuged at 10,000 rpm for 10 minutes using a Nanosep $10 \mathrm{k}$ membrane filtration centrifuge tube (Pall, Life Sciences, USA). The drug concentration in the lower compartment was determined by fluorescence spectrophotometry as previously described. The particles in the upper compartment were resuspended in $0.1 \mathrm{~mL}$ of the PBS solution and transferred back to the tube. The cumulative DOX release was determined from (2):

$$
\begin{aligned}
& \text { Cumulative DOX Released (\%) } \\
& =\frac{\text { Amount of DOX release }}{\text { Initial amount DOX }} \times 100 .
\end{aligned}
$$

2.7. Cells and Culture. Human breast cancer cells (MCF-7) were obtained from the National Center for Genetic Engineering and Biotechnology (BIOTEC) (Bangkok, Thailand), while mouse fibroblast cells (L929) were provided by the Tissue Laboratory, King Chulalongkorn Memorial Hospital (Bangkok, Thailand). Both cell lines were maintained in DMEM medium supplemented with $10 \%(\mathrm{v} / \mathrm{v})$ FBS, 100 units $/ \mathrm{mL}$ penicillin, and $100 \mu \mathrm{g} / \mathrm{mL}$ streptomycin in a humidified atmosphere containing $5 \% \mathrm{CO}_{2}$ at $37^{\circ} \mathrm{C}$.

2.8. Cytotoxicity Studies of Core-Shell Nanoparticles. The cytotoxicity of the core-shell nanoparticles was investigated in two parts. First, the relative toxicity of the nanoparticles without DOX was determined. L929 cells at a density of 2,000 cells/well were grown on 96-well microplates and were treated with chitosan nanoparticles and folate-conjugated chitosan nanoparticles for 3 days. The cell viability was determined using the MTT assay following the standard procedure (Molecular Probes, V-13154). The absorbance of formazan was measured at $570 \mathrm{~nm}$ (Infinite 200 Tecan, Austria). Results were presented as percentage of control cells not exposed to the nanoparticles.

In the second part, the efficacy of the nanoparticles as delivery vectors was evaluated. This study was also carried out in 96-well microplates. MCF-7 cells at a density of 5,000 cells/well were exposed to various concentrations of free DOX, DOX-loaded chitosan nanoparticles, and DOX-loaded folate-conjugated chitosan nanoparticles for 3 days. The cell viability was measured using the MTT assay, and the relative toxicity was obtained using nontreated MCF-7 cells as control.
2.9. Drug Accumulation Study. MCF-7 cells at a density of 50,000 cells/well were allowed to grow until confluence in 96well culture plates. Twenty $\mathrm{mg} / \mathrm{mL}$ of free DOX, DOX-loaded chitosan nanoparticles, and DOX-loaded folate-conjugated chitosan nanoparticles were added to each culture well. At each predetermined time, spent medium was removed and replaced with $50 \mu \mathrm{l}$ of $70 \%$ ethanol [38]. The samples were briefly sonicated for 30 seconds to ensure complete cell lysis. The concentrations of doxorubicin in the samples were measured using a fluorescence spectrophotometer (Infinite 200 Tecan, Austria). To monitor cellular uptake of the drug, MCF-7 cells were cultured on glass cover slips and incubated with DOX-loaded nanoparticles up to 24 hours. The cover slips were viewed at 1,2 , and 24 hours after the initial treatment under a fluorescence microscope $\left(\lambda_{\text {excitation }}=465 \pm\right.$ $15 \mathrm{~nm}$ and $\lambda_{\text {emission }}=590 \mathrm{~nm}$; Olympus BX60, Center Valley, PA). Images were acquired using a $20 \times$ objective lens.

2.10. Statistical Analysis. All experiments were performed at least in triplicate within two independent experiments. Data are reported as mean \pm standard deviation. An unpaired Student's $t$-test was used for data analysis. A statistically significant difference was defined at the $95 \%$ confidence level.

\section{Results and Discussion}

The preparation of DOX-loaded folate-conjugated chitosan nanoparticles in a core-shell structure is illustrated in Figure 1. The core consists of a spherical pluronic and SDS micelle formed via self-assembly, which is a spontaneous process at or above the critical micelle concentration (CMC) of around $0.1 \%(\mathrm{w} / \mathrm{v})[7,20,34,39]$. Then, a layer of chitosan was coated on the surface of these nanocarriers using electrostatic interactions between the positively charged chitosan and the negatively charged SDS on the surface of pluronic SDS mixed micelles. The chitosan shells can protect the enclosed bioactive compounds from natural degradation, such as hydrolysis, thereby prolonging its activity in the systemic circulation $[17,19,40]$. In addition to stability improvement, the shell can be modified to incorporate ligands such as folate for targeting drug delivery $[8,28,32,33]$. According to our previous study, folate was successfully conjugated to chitosan using the method described in the previous section. The conjugation was confirmed by its FT-IR spectrum, which showed the presence of a CONH bond (see Supplementary A in Supplementary Material available online at doi:10.1155/ 2012/593878). The binding efficiency was approximately $60.1 \pm 4.4 \%[41]$.

Once at target cells, the uptake of the nanoparticles can occur through an endocytosis process [42]. The chitosan shell is then degraded by lysozyme, which is normally present inside cellular lysosomes, allowing the breakdown of the micelles and the release of DOX to nucleus [12, 37, 43]. This method is advantageous because it is simple, mild, and straightforward and it can be applicable to a wide range of bioactive compounds.

3.1. Evaluation of Drug Encapsulation Efficiency. The study of DOX encapsulation efficiency was carried out to determine 


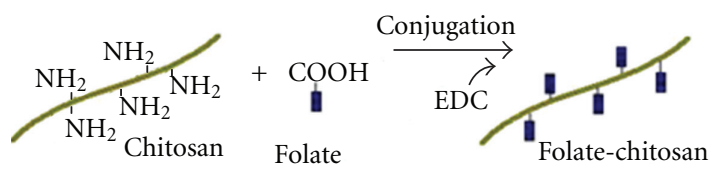

(a)

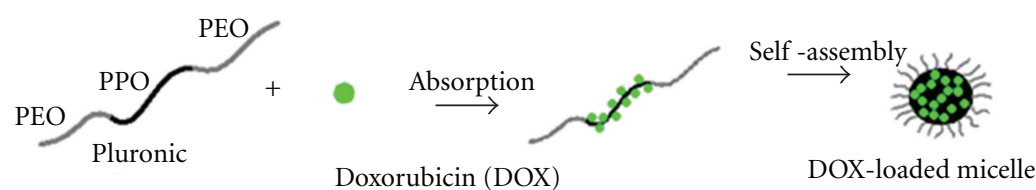

(b)

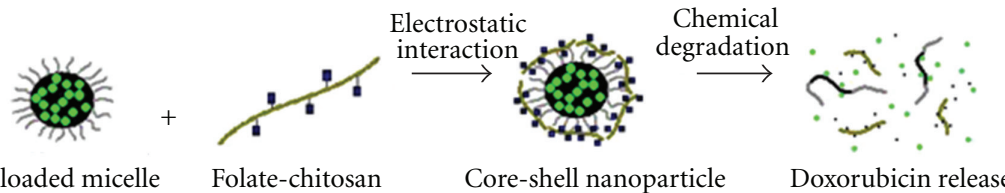

(c)

FIGURE 1: Procedure for the synthesis of DOX-loaded folate-conjugated chitosan core-shell nanoparticles. (a) Conjugation of folate-chitosan, (b) self-assembly of DOX-encapsulated micelles, (c) core-shell nanoparticles prepared by an electrostatic interaction.

an appropriate concentration of pluronic. In this study, pluronic concentrations were varied between 0.25 and $10 \%$ $(\mathrm{w} / \mathrm{v})$, while the concentration of DOX was kept constant. After the evaporation step, DOX was physically entrapped in the hydrophobic interior of the micelles [6]. Excess DOX in the supernatant was removed and used to calculate the encapsulation efficiency according to (1). The encapsulation efficiencies of DOX in various concentrations of pluronic micelles are shown in Figure 2. When pluronic concentration was increased from 1.25 to $5 \%(\mathrm{w} / \mathrm{v})$, the drug encapsulation efficiency increased from $35.2 \pm 1.0$ to $58.1 \pm 4.7 \%$, possibly due to an increase in the hydrophobic domain of the micelles. As a result, more DOX could be encapsulated [44]. Interestingly, the difference in the DOX encapsulation efficiencies between $5 \%(\mathrm{w} / \mathrm{v})$ and $10 \%(\mathrm{w} / \mathrm{v})$ pluronic was not significant $(P>0.05)$, indicating that nanoparticle saturation was reached. The encapsulation efficiency observed in this study is reasonably high for a mild solvent evaporation method. Missirlis et al. [10,22] and Kwon and Kataoka [21] reported the encapsulation efficiencies of DOX in pluronic nanogel to be approximately $10-50 \%$ when $\mathrm{CHCl}_{3}$ was used as solvent. The previous report has shown that the partition coefficient of doxorubicin between water and pluronic micelles is 0.44 at neutral $\mathrm{pH}$ [45]. Thus, only a certain amount of DOX is available to be encapsulated, resulting in the limit of the encapsulation efficiency by this physical entrapment procedure. We believe that the encapsulation efficiency of DOX in pluronic micelles observed in this study may possibly be the highest efficiency achievable by this method. To improve the efficiency, doxorubicin can be conjugated with pluronic using a chemical linker [46]. However, this method would be more complicated, and the release rate would tend to be slower. Since $5 \%(\mathrm{w} / \mathrm{v})$ of pluronic micelles gave the highest encapsulation efficiency, this condition was selected for further studies.

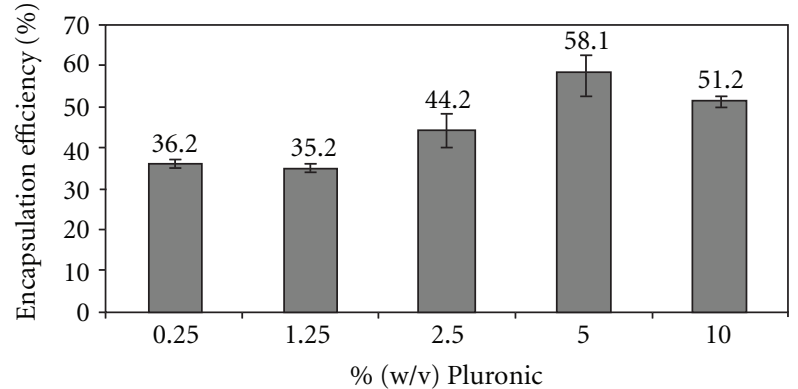

FIGURE 2: Encapsulation efficiencies of DOX in the micelles prepared from various Pluronic F127 concentrations.

3.2. Particle Size and Zeta Potential of Core-Shell Nanoparticles. Particle size and zeta potential of both micelles and DOX-loaded micelles were measured using a Zetasizer as shown in Table 1. The average micelle size remained the same after DOX encapsulation $(33.7 \pm 1.0 \mathrm{~nm}$ and $32.6 \pm 2.1 \mathrm{~nm}$ for the average size of pluronic micelles with and without DOX, resp.). The zeta potential of pluronic micelles in the absence and presence of DOX were $-4.8 \pm 1.3$ and $-4.4 \pm 0.8 \mathrm{mV}$. The zeta potentials, however, showed that these micelles were not stable and could easily coagulate, which can be problematic for drug delivery applications.

To solve this problem, incubating micelles with sodium dodecyl sulfate (SDS) was required. SDS is an anionic surfactant that can interact with pluronic micelles by binding to the hydrophobic core, followed by interacting with hydrophilic corona parts [47]. Consequently, the micelles were packed more tightly [17], decreasing their average size to approximately $23 \mathrm{~nm}$, as shown in Table 1 . With increasing concentration of surfactant, the average micelle size in the solution decreases [48]. In addition, both the sulfate group of SDS 
TABle 1: Particle sizes and zeta potentials of pluronic micelles, chitosan nanoparticles, and folate-conjugated chitosan nanoparticles in the absence and presence of DOX.

\begin{tabular}{|c|c|c|c|c|}
\hline Condition & Particle diameter (nm) & $\%$ intensity & Zeta potential $(\mathrm{mV})$ & $\mathrm{pH}$ \\
\hline \multicolumn{5}{|l|}{ Nanoparticles } \\
\hline (i) Micelles & $32.6 \pm 2.1$ & 81.9 & $-4.8 \pm 1.3$ & 7.12 \\
\hline (ii) Micelles with SDS & $22.9 \pm 0.3$ & 73.7 & $-11.4 \pm 0.6$ & 7.06 \\
\hline (iii) Chitosan nanoparticles & $34.1 \pm 7.1$ & 94.4 & $6.2 \pm 3.6$ & 4.70 \\
\hline (iv) Folate-conjugated chitosan nanoparticles & $41.3 \pm 6.5$ & 96.3 & $8.3 \pm 1.1$ & 4.74 \\
\hline \multicolumn{5}{|l|}{ DOX-loaded Nanoparticles } \\
\hline (i) DOX-loaded micelles & $33.7 \pm 1.0$ & 53.1 & $-4.4 \pm 0.8$ & 7.07 \\
\hline (ii) DOX-loaded micelles with SDS & $20.3 \pm 1.0$ & 55.6 & $-3.8 \pm 0.8$ & 7.03 \\
\hline (iii) DOX-loaded chitosan nanoparticles & $30.9 \pm 1.7$ & 96.3 & $10.6 \pm 2.9$ & 4.69 \\
\hline (iv) DOX-loaded folate-conjugated chitosan nanoparticles & $37.4 \pm 2.0$ & 94.9 & $12.9 \pm 2.3$ & 4.71 \\
\hline
\end{tabular}

and the denser $\mathrm{OH}^{-}$group on the surface of the micelles contribute to more negative zeta potential $(-11.4 \pm 0.6 \mathrm{mV})$, making the micelles relatively more stable. Note that the addition of SDS, however, caused scattered micelle sizes, as shown by lower particle intensity in Table 1 . This observation was similar to the results from Hecht and Hoffmann [47]. Particles larger than $200 \mathrm{~nm}$ in diameter were removed using a membrane filter prior to coating the particles with chitosan or folate-conjugated chitosan.

To form core-shell nanoparticles, either chitosan or folate-conjugated chitosan was added to the pluronic SDS micelle solution at the same concentration, allowing for the deposition of chitosan or folate-conjugated chitosan layer on the pluronic micelles using SDS as a linker [17, 19, 49, 50]. As expected, the average particles sizes were increased approximately by $10-18 \mathrm{~nm}$. The average size of folate-conjugated chitosan particles was slightly larger than that of chitosan particles, possibly due to the conjugation of folate. The zeta potentials of the coated particles indicated that the surface charges were positive as a result of $\mathrm{NH}_{3}{ }^{+}$groups of chitosan. This result confirms our hypothesis that chitosan can be layered on pluronic micelles via an electrostatic interaction. In addition, the high zeta potential of chitosancoated pluronic micelles implies that the core-shell structure improved the stability of the nanoparticles.

The size distribution of DOX-loaded micelles coated with folate-conjugated chitosan is shown in Supplementary B. The sizes of most particles were uniformly distributed over the range of 15 to $90 \mathrm{~nm}$. Approximately 5\% of the particles had sizes over $1 \mu \mathrm{m}$ in diameter and were removed by filtration.

To be more certain, a morphological characterization of the folate-conjugated chitosan nanoparticles was carried out. Transmission electron microscopy (TEM) images of the DOX-loaded core shell nanoparticles are shown in Figure 3. The nanoparticles were shown to be spherical in shape with uniform sizes. The enlarged image of a single nanoparticle clearly reveals a core-shell structure of the particle. The darker shaded area was believed to be the pluronic micelle core, while the lighter shaded area was most likely the shell of folate-conjugated chitosan.
3.3. In Vitro Doxorubicin Release from Core-Shell Nanoparticles. The studies of DOX released from pluronic micelles, chitosan nanoparticles, and folate-conjugated chitosan nanoparticles were carried out in a PBS solution in the presence of lysozyme over 4 days. Lysozyme is an enzyme naturally present in all cell types and is able to hydrolyze $\beta$ - $(1,4)$ glycosidic bonds, causing the breakdown of a chitosan shell [36, $37,51]$. As shown in Figure 4, almost $100 \%$ of encapsulated DOX was steadily released from pluronic micelles within 24 hours, while the core-shell structure nanoparticles show much slower and sustained release profiles. This result indicates that the chitosan layer significantly extended the halflife of the drug-encapsulated nanocarriers which is important in clinical practice.

The release profiles of DOX from the core-shell nanoparticles could be separated into two phases. The first profile is the initial burst release of $19.6 \%$ and $22.4 \%$ of DOX from chitosan and folate-conjugated chitosan nanoparticles, respectively, in the first 24 hours. This is possibly due to the drug molecules dispersing close to the polymer surface. In the second phase, a constant sustained release of the drug after 24 hours was observed, resulting from the diffusion of the drug through the polymer wall, as well as the enzymatic degradation of chitosan $[1,9]$. The release profiles observed in this study are quite similar to those obtained from chitosan nanoparticles elsewhere $[11,15,52]$, in which the kinetics often follow the first-order rate law [53].

3.4. Biocompatibility Test of Core-Shell Nanoparticles. Since the particles' toxicity has an essential impact on their applications in the drug delivery field, preliminary biocompatibility evaluation is necessary. According to International Standard Organization (ISO 10993), a direct contact method where cells are directly in contact with a test material is more appropriate than an indirect contact method to assess the cytotoxicity of nanoparticles. Mouse connective tissue fibroblasts (L929) were chosen for this study, as they are a standard model for in vitro toxicity testing and recommended by many institutions. L929 cells were treated with pluronic micelles, chitosan nanoparticles, and folate-conjugated 


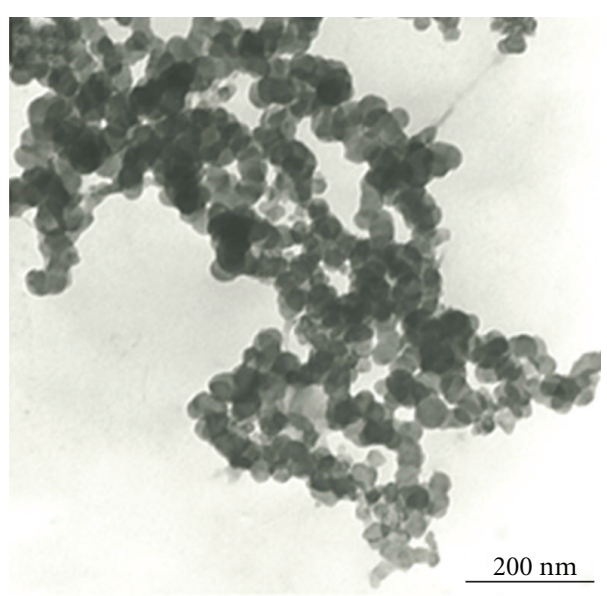

(a)

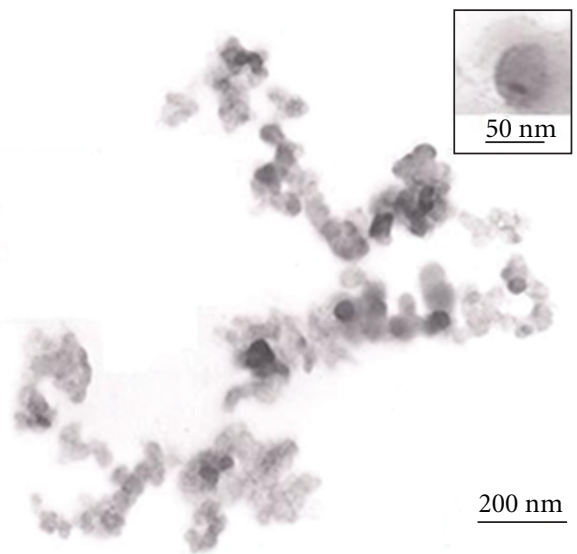

(b)

FIgURE 3: Transmission electron microscopy (TEM) images of (a) blank pluronic micelles and (b) folate-modified chitosan core-shell nanoparticles. The inset image is the enlarged image of a single nanoparticle present in the previous image: dark color core of pluronic and light-gray shell of folate-modified chitosan.

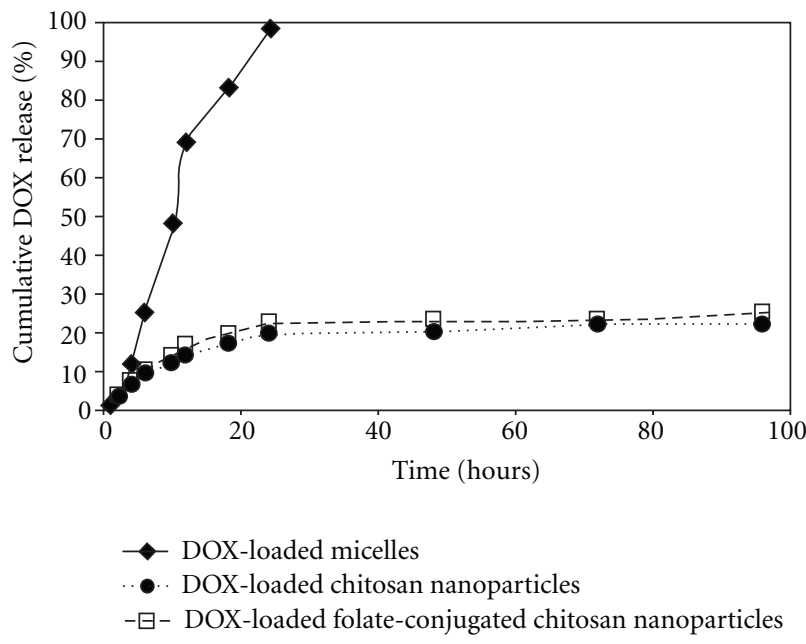

FIGURE 4: Release profiles of DOX from pluronic micelles, chitosan core-shell nanoparticles, and folate-conjugated chitosan core-shell nanoparticles in $\mathrm{PBS}, \mathrm{pH} 7.4$, at $37^{\circ} \mathrm{C}$.

chitosan nanoparticles in the absence of DOX at various equivalent concentrations for 3 days. The cell viability was expressed in percent compared to the control, which was not exposed to any particles.

As shown in Figure 5, both chitosan core-shell and folateconjugated chitosan core-shell nanoparticles showed comparable toxicity $(P>0.05)$. The cell viability was not affected by these particles at concentrations lower than $1 \mathrm{mg} / \mathrm{mL}$ but showed progressive decline to about $60-70 \%$ when exposed to $10 \mathrm{mg} / \mathrm{mL}$ concentration of the particles. This result suggested that both chitosan and folate-conjugated chitosan core-shell nanoparticles can be considered safe as drug delivery vectors.

3.5. Cellular Uptake of DOX by MCF-7 Cells. The study of intracellular uptake of DOX by MCF-7 cells was conducted to

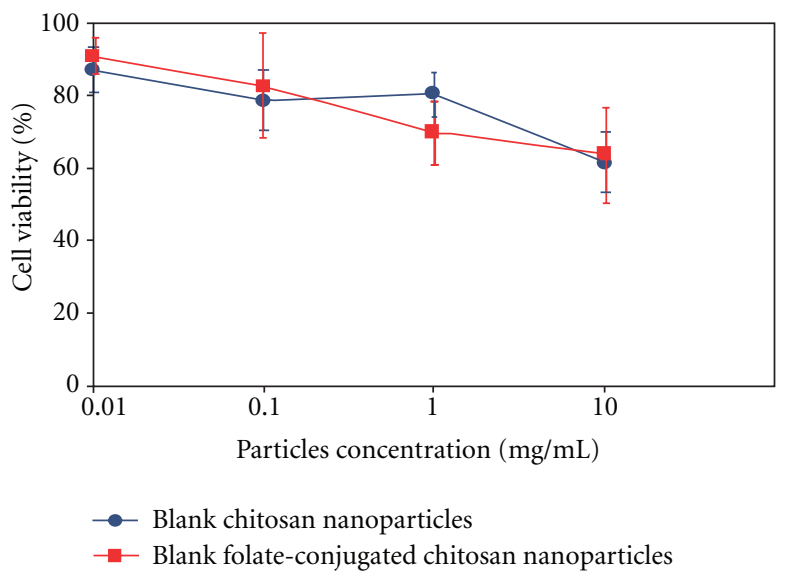

FIGURE 5: Cytotoxicity profiles of blank chitosan core-shell nanoparticles and blank folate-conjugated chitosan core-shell nanoparticles on L929 cells, measured by the MTT assay. Cell viability is expressed as mean \pm S.D. $(n=6)$. The cells were incubated in different equivalent concentrations of these particles, and the cell viability was determined by the MTT assay.

demonstrate that the cellular uptake of the nanoparticles was possible and was controlled over time. The amount of DOX taken up by MCF-7 cells from different formulations for up to 24 hours was analyzed by fluorescence spectrophotometry. As shown in Table 2, free DOX accumulated rapidly in the cells via passive diffusion through the cell membrane. More than $80 \%$ of the DOX was taken up by the cells within 2 hours, and almost 100\% uptake could be achieved within 24 hours. In contrast, a much slower cellular uptake rate was observed from DOX-loaded nanoparticles. Only 15\% and $26 \%$ of the DOX from chitosan and folate-conjugated chitosan nanoparticles, respectively, had accumulated in the cells at 24 hours. Nonetheless, the cellular uptake of folate-conjugated chitosan nanoparticles was about twice as much as that 
TABle 2: Cellular uptake of DOX by MCF-7 cells treated with free DOX, DOX-loaded chitosan nanoparticles, and DOX-loaded folateconjugated chitosan nanoparticles for up to 24 hours. Results are presented as percent of the initial concentration of DOX.

\begin{tabular}{lcccccc}
\hline \multirow{2}{*}{ Conditions } & \multicolumn{5}{c}{ Cellular DOX uptake (\% of its initial concentration) } \\
& $1 \mathrm{~h}$ & $2 \mathrm{~h}$ & $6 \mathrm{~h}$ & $12 \mathrm{~h}$ & $18 \mathrm{~h}$ & $24 \mathrm{~h}$ \\
\hline Free DOX & $26.8 \pm 0.58$ & $80 \pm 1.3$ & $74.7 \pm 2.1$ & $98.0 \pm 1.7$ & $93.7 \pm 2.7$ & $102.8 \pm 4.4$ \\
DOX-loaded micelles coated with chitosan & $0.5 \pm 0.1$ & $0.6 \pm 0.2$ & $2.7 \pm 0.6$ & $4.5 \pm 1.0$ & $11.2 \pm 0.8$ & $14.7 \pm 1.5$ \\
DOX-loaded micelles coated with modified chitosan & $0.6 \pm 0.4$ & $0.7 \pm 0.6$ & $10.9 \pm 0.7$ & $12.7 \pm 0.7$ & $21.1 \pm 1.9$ & $26.3 \pm 1.9$ \\
\hline
\end{tabular}

of chitosan nanoparticles. The cellular uptake of the chitosan nanoparticles, which have no targeting moiety on the surface, was probably mediated by nonspecific adsorptive endocytosis $[54,55]$. The positive charge of the chitosan nanoparticles could be easily attracted by negatively charged cell membranes [16], allowing the particles to be taken into the cells. Unlike the chitosan nanoparticles, the conjugation of the folate ligand on folate-conjugated chitosan nanoparticles enabled the cellular uptake to occur via a receptor-mediated endocytosis process [32]. Several studies have shown that receptor-mediated endocytosis is significantly more efficient than nonspecific adsorptive endocytosis in transporting macromolecules into cells [27, 30, 42]. As a result, more DOX was accumulated in the cells exposed to folate-conjugated chitosan nanoparticles containing DOX than in those cells treated with DOX-loaded chitosan nanoparticles.

Two possible mechanisms of cellular DOX uptake from nanoparticles have been suggested: (1) DOX is released from the nanoparticles outside the cells, or (2) DOX is carried by the nanoparticles and released inside the cells [56]. To assess the uptake of the nanoparticles by the cells, the fluorescence micrographs of MCF-7 cells incubated with free DOX were compared to those of cells exposed to folate-conjugated chitosan nanoparticles containing DOX at 1, 2, and 24 hours (Figure 6). At earlier times, no red fluorescence was detected in either MCF-7 cultures (Figure 6(a) and 6(d)). Within 2 hours of incubation, free DOX was localized within the cytoplasm and the nucleus (Figure 6(b)), while the red fluorescence of DOX encapsulated in thefolate-conjugated chitosan nanoparticles was only detected around the edge of the cells, indicating the binding of the nanoparticles on the cell membrane (Figure 6(e)). The intracellular localization of the nanoparticles could be visualized only after 24 hours of incubation, as some of the cell nuclei displayed strong red fluorescence (Figure 6(f)) similar to those incubated with free DOX for 24 hours (Figure 6(c)). A much longer incubation time was required for the nanoparticles to be internalized. These results suggest that the nanoparticles were initially adhered to the cell membrane and bound to the folate receptors, overexpressed on the cell surface, before being taken into the cells via endocytosis and later degraded intracellularly to release the drug. This finding is consistent with the results previously reported by several studies $[11-13,16$, 23].

3.6. Cytotoxicity of Core-Shell Nanoparticles. The cytotoxicity profiles of blank and DOX-loaded nanoparticles against MCF-7 cells are shown in Figure 7. As expected, the blank nanoparticles exhibited negligible toxicity towards MCF-7 cells, while the cytotoxicity to the cells increased with increasing concentration of the drug-loaded nanoparticles. There was no significant difference between the cytotoxicity profiles of DOX-loaded chitosan nanoparticles and that of folateconjugated chitosan nanoparticles containing DOX. Since both chitosan and folate-conjugated chitosan nanoparticles were shown to be nontoxic, any toxicity observed in this study was likely attributable to the DOX in the nanoparticles. The $\mathrm{IC}_{50}$ of DOX-loaded chitosan nanoparticles and folateconjugated chitosan nanoparticles containing DOX was calculated to be 34 and $26 \mathrm{ng} / \mathrm{mL}$, respectively, whereas the $\mathrm{IC}_{50}$ of free DOX was experimentally determined to be $386 \mathrm{ng} / \mathrm{mL}$ (unpublished result). The $\mathrm{IC}_{50}$ values of DOX-loaded nanoparticles were approximately 10 times lower than that of free DOX, indicating a higher therapeutic effect of the drugloaded nanoparticles. The lower $\mathrm{IC}_{50}$ might be attributed to the longer duration of the treatment ( 3 days as opposed to the 24 hours used in the intracellular uptake study), which was necessary to allow more nanoparticles to be internalized in order to release a sufficient concentration of DOX within its therapeutic range. The study by Yoo and Park [23] has offered another possible explanation: free DOX might have been outfluxed through the P-glycoprotein (Pgp) pumps present in the cancer cell membrane, causing a decrease in the intracellular concentration of DOX. Unlike free DOX, DOXloaded nanoparticles were intracellularly delivered into the cells by endocytosis, which by-passed the Pgp pump. As a result, a greater amount of DOX could remain inside the cells to cause a cytotoxic effect. More thorough studies are needed to further understand the mechanism of toxicity of the drugloaded nanoparticles against the cancer cells.

Unfortunately, there was no significant difference between the cytotoxicity profiles of DOX-loaded chitosan and folate-conjugated chitosan nanoparticles. It was possible that the number of ligands on the surface of the nanoparticles might not be sufficient to demonstrate the advantage of having folate targeting. The ligand conjugation method should be modified to increase the number of ligand moieties. In addition, in order to observe folate-induced specificity, comparisons of the cytotoxicity of these nanoparticles on a cancer and non-cancer cell line would be useful.

\section{Conclusion}

The core-shell nanoparticles for DOX delivery were successfully formed using a self-assembled pluronic F127 micelle as a core coated with either chitosan or folate-conjugated chitosan via an electrostatic interaction. The presence of a chitosan layer on the nanoparticles was confirmed by the change 
Free DOX

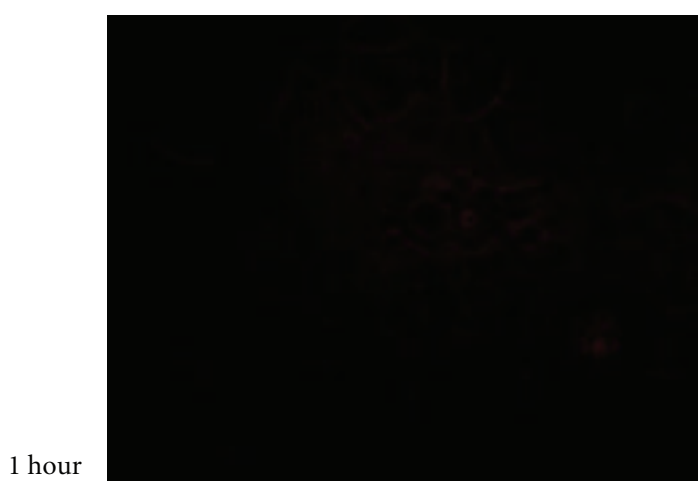

(a)

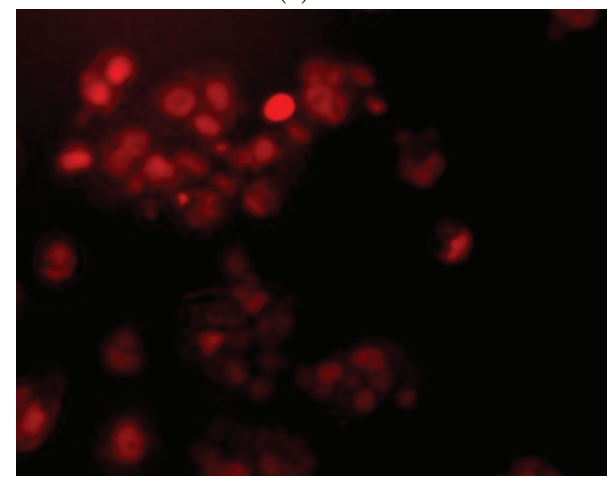

(c)

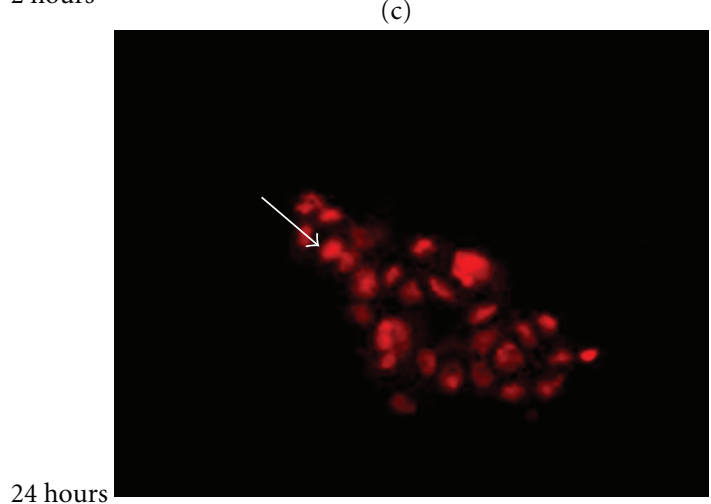

(e)

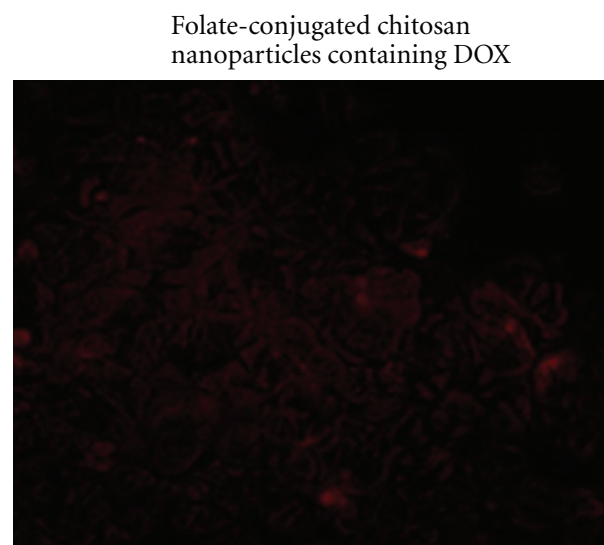

(b)

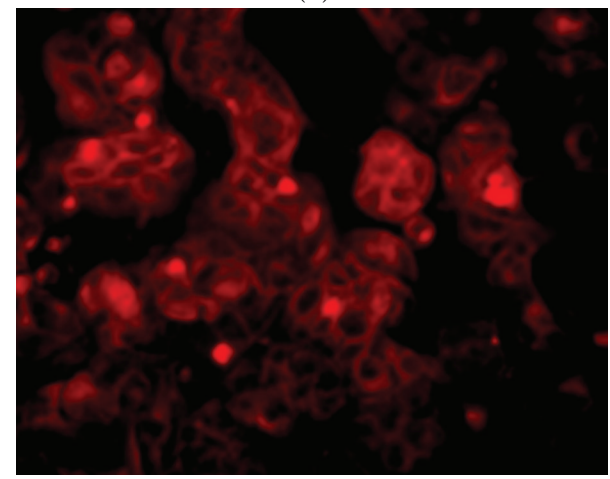

(d)

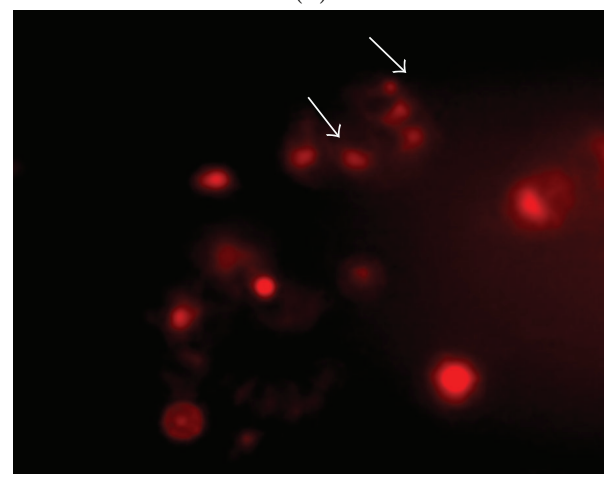

(f)

FIGURE 6: Fluorescence images of cellular distribution of free DOX ((a) 1 hour, (b) 2 hour, and (c) 24 hour incubation) and DOX-loaded folate-conjugated chitosan core-shell nanoparticles ((d) 1 hour, (e) 2 hour, and (f) 24 hour incubation) in MCF-7 cells. The arrows indicate the accumulation of DOX at the cell nuclei. Magnifications: 20X.

in its zeta potential and morphologically characterized by TEM images. We were able to encapsulate considerably high concentrations of DOX inside the nanoparticles through a simple physical entrapment method. These particles demonstrated an initial burst release, and later a sustained release, of DOX. In addition, the fluorescence images showed that these nanoparticles can be delivered and then intracellularly release DOX in MCF-7 by an endocytosis mechanism. The uptake rate of the folate-conjugated chitosan nanoparticles was relatively faster than that of the chitosan nanoparticles, possibly due to the more effective folate-mediated endocytosis. Both types of the nanoparticles exhibited similar toxicity profiles against MCF-7 cells. Our results indicated that the lower cell viability corresponded to a higher DOX concentration and longer incubation time. Therefore, we believe that these core-shell nanoparticles are promising as delivery vectors for the treatment of tumor tissues, particularly breast cancer, for which folate receptors are overexpressed.

\section{Acknowledgments}

This work was supported by the Research, Development and Engineering (RD\&E) Fund through the National Nanotechnology Center (NANOTEC), the National Science and Technology Development Agency (NSTDA), Thailand 


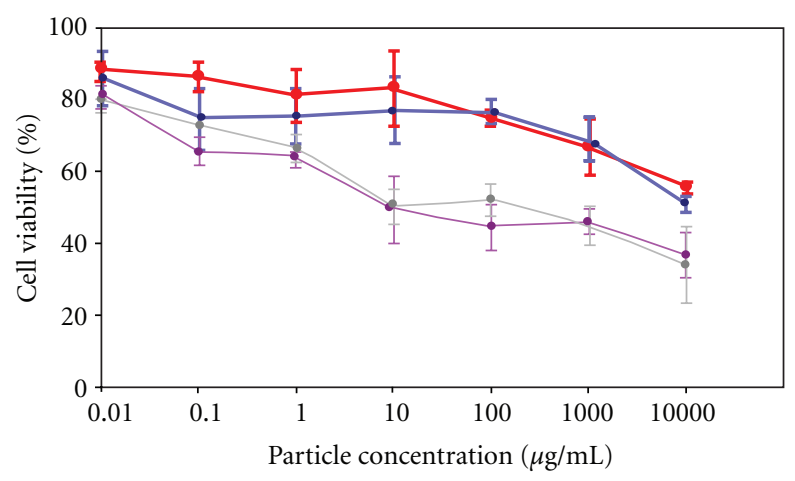

- Blank folate-conjugated chitosan nanoparticles

- Blank chitosan nanoparticles

- DOX-loaded folate-conjugated chitosan nanoparticles

$\rightarrow$ DOX-loaded chitosan nanoparticles

Figure 7: Cytotoxicity $\left(\mathrm{IC}_{50}\right)$ of blank pluronic micelles, blank folate-conjugated chitosan nanoparticles, DOX-loaded micelles, DOX-loaded chitosan nanoparticles, and DOX-loaded folate-conjugated chitosan nanoparticles against MCF-7 cells. The cells were incubated in different concentrations of those formulations for 3 days. The cell viability was compared to the control in which the cells were not exposed to any particles and was expressed as mean \pm S.D.

(Project no. NN-B-22-EN6-e21-51-9), the Higher Educational Research Promotion and National Research University Project of Thailand, the Office of the Higher Education Commission, the Thailand Graduate Institute of Science and Technology (TGIST), and the National Science and Technology Development Agency (TG-55-20-51-038). The authors do not have a direct financial relation with Pluronic and BASF that might lead to a conflict of interest for any of the authors.

\section{References}

[1] M. A. Edward, "Unresolved structure-activity relationships in anthracycline analogue development," in Anthracycline Antibiotics: New Analogues, Methods of Delivery and Mechanisms of Action, P. Waldemar, Ed., pp. 1-13, American Chemical Society, Washington, DC, USA, 1995.

[2] S. Lee, M. Baek, H. Y. Kim, J. H. Ha, and D. I. Jeoung, "Mechanism of doxorubicin-induced cell death and expression profile analysis," Biotechnology Letters, vol. 24, no. 14, pp. 1147-1151, 2002.

[3] I. Müller, A. Jenner, G. Bruchelt, D. Niethammer, and B. Halliwell, "Effect of concentration on the cytotoxic mechanism of doxorubicin-apoptosis and oxidative DNA damage," Biochemical and Biophysical Research Communications, vol. 230, no. 2, pp. 254-257, 1997.

[4] E. Andreopoulou, D. Gaiotti, E. Kim et al., "Pegylated liposomal doxorubicin HCL (PLD; Caelyx/Doxil): experience with long-term maintenance in responding patients with recurrent epithelial ovarian cancer," Annals of Oncology, vol. 18, no. 4, pp. 716-721, 2007.

[5] H. D. Han, A. Lee, C. K. Song et al., "In vivo distribution and antitumor activity of heparin-stabilized doxorubicin-loaded liposomes," International Journal of Pharmaceutics, vol. 313, no. 1-2, pp. 181-188, 2006.

[6] V. Alakhov, E. Klinski, S. Li et al., "Block copolymer-based formulation of doxorubicin. From cell screen to clinical trials," Colloids and Surfaces B, vol. 16, no. 1-4, pp. 113-134, 1999.

[7] P. K. Sharma and S. R. Bhatia, "Effect of anti-inflammatories on Pluronic $囚$ F127: micellar assembly, gelation and partitioning," International Journal of Pharmaceutics, vol. 278, no. 2, pp. 361-377, 2004.

[8] H. S. Yoo and T. G. Park, "Folate-receptor-targeted delivery of doxorubicin nano-aggregates stabilized by doxorubicin-PEGfolate conjugate," Journal of Controlled Release, vol. 100, no. 2, pp. 247-256, 2004.

[9] Y. I. Cho, S. Park, S. Y. Jeong, and H. S. Yoo, "In vivo and in vitro anti-cancer activity of thermo-sensitive and photocrosslinkable doxorubicin hydrogels composed of chitosandoxorubicin conjugates," European Journal of Pharmaceutics and Biopharmaceutics, vol. 73, no. 1, pp. 59-65, 2009.

[10] D. Missirlis, R. Kawamura, N. Tirelli, and J. A. Hubbell, "Doxorubicin encapsulation and diffusional release from stable, polymeric, hydrogel nanoparticles," European Journal of Pharmaceutical Sciences, vol. 29, no. 2, pp. 120-129, 2006.

[11] K. A. Janes, M. P. Fresneau, A. Marazuela, A. Fabra, and M. J. Alonso, "Chitosan nanoparticles as delivery systems for doxorubicin," Journal of Controlled Release, vol. 73, no. 2-3, pp. 255-267, 2001.

[12] P. S. Lai, P. J. Lou, C. L. Peng et al., "Doxorubicin delivery by polyamidoamine dendrimer conjugation and photochemical internalization for cancer therapy," Journal of Controlled Release, vol. 122, no. 1, pp. 39-46, 2007.

[13] Y. Lee, S. Y. Park, H. Mok, and T. G. Park, "Synthesis, characterization, antitumor activity of pluronic mimicking copolymer micelles conjugated with doxorubicin via acid-cleavable linkage," Bioconjugate Chemistry, vol. 19, no. 2, pp. 525-531, 2008.

[14] F. Caruso, "Engineering of core-shell particles and hollow capsules," in Nano-Surface Chemistry, M. Rosoff, Ed., chapter 14, Marcel Dekker, New York, NY, USA, 2001.

[15] S. Papadimitriou, D. Bikiaris, K. Avgoustakis, E. Karavas, and M. Georgarakis, "Chitosan nanoparticles loaded with dorzolamide and pramipexole," Carbohydrate Polymers, vol. 73, no. 1, pp. 44-54, 2008.

[16] S. K. Sahu, S. K. Mallick, S. Santra, T. K. Maiti, S. K. Ghosh, and P. Pramanik, "in vitro evaluation of folic acid modified carboxymethyl chitosan nanoparticles loaded with doxorubicin for targeted delivery," Journal of Materials Science, vol. 21, no. 5, pp. 1587-1597, 2010.

[17] Z. Lei and S. Bi, "The silica-coated chitosan particle from a layer-by-layer approach for pectinase immobilization," Enzyme and Microbial Technology, vol. 40, no. 5, pp. 1442-1447, 2007.

[18] N. Pimpha, U. Rattanonchai, S. Surassmo, P. Opanasopit, C. Rattanarungchai, and P. Sunintaboon, "Preparation of PMMA/acid-modified chitosan core-shell nanoparticles and their potential as gene carriers," Colloid and Polymer Science, vol. 286, no. 8-9, pp. 907-916, 2008.

[19] B. S. Kim, C. S. Kim, and K. M. Lee, "The intracellular uptake ability of chitosan-coated Poly (D,L-lactideco-glycolide) nanoparticles," Archives of Pharmacal Research, vol. 31, no. 8, pp. 1050-1054, 2008. 
[20] J. J. Escobar-Chávez, M. López-Cervantes, A. Naïk, Y. N. Kalia, D. Quintanar-Guerrero, and A. Ganem-Quintanar, "Applications of thermo-reversible pluronic F-127 gels in pharmaceutical formulations," Journal of Pharmacy and Pharmaceutical Sciences, vol. 9, no. 3, pp. 339-358, 2006.

[21] G. S. Kwon and K. Kataoka, "Block copolymer micelles as long-circulating drug vehicles," Advanced Drug Delivery Reviews, vol. 16, no. 2-3, pp. 295-309, 1995.

[22] D. Missirlis, N. Tirelli, and J. A. Hubbell, "Amphiphilic hydrogel nanoparticles. Preparation, characterization, and preliminary assessment as new colloidal drug carriers," Langmuir, vol. 21, no. 6, pp. 2605-2613, 2005.

[23] H. S. Yoo and T. G. Park, "Folate receptor targeted biodegradable polymeric doxorubicin micelles," Journal of Controlled Release, vol. 96, no. 2, pp. 273-283, 2004.

[24] K. H. Bae, Y. J. Ha, C. Kim, K. R. Lee, and T. G. Park, "Pluronic/chitosan shell cross-linked nanocapsules encapsulating magnetic nanoparticles," Journal of Biomaterials Science, vol. 19, no. 12, pp. 1571-1583, 2008.

[25] A. M. De Campos, Y. Diebold, E. L. S. Carvalho, A. Sánchez, and M. J. Alonso, "Chitosan nanoparticles as new ocular drug delivery systems: in vitro stability, in vivo fate, and cellular toxicity," Pharmaceutical Research, vol. 21, no. 5, pp. 803-810, 2004.

[26] J. A. Ko, H. J. Park, S. J. Hwang, J. B. Park, and J. S. Lee, "Preparation and characterization of chitosan microparticles intended for controlled drug delivery," International Journal of Pharmaceutics, vol. 249, no. 1-2, pp. 165-174, 2002.

[27] J. Liu, C. Kolar, T. A. Lawson, and W. H. Gmeiner, “Targeted drug delivery to chemoresistant cells: folic acid derivatization of FdUMP enhances cytotoxicity toward 5-fu-resistant human colorectal tumor cells," Journal of Organic Chemistry, vol. 66, no. 17 , pp. 5655-5663, 2001.

[28] S. Mansouri, Y. Cuie, F. Winnik et al., "Characterization of folate-chitosan-DNA nanoparticles for gene therapy," Biomaterials, vol. 27, no. 9, pp. 2060-2065, 2006.

[29] E. I. Sega and P. S. Low, "Tumor detection using folate receptor-targeted imaging agents," Cancer and Metastasis Reviews, vol. 27, no. 4, pp. 655-664, 2008.

[30] S. J. Yang, F. H. Lin, K. C. Tsai et al., "Folic acid-conjugated chitosan nanoparticles enhanced protoporphyrin IX accumulation in colorectal cancer cells," Bioconjugate Chemistry, vol. 21, no. 4, pp. 679-689, 2010.

[31] P. Chan, M. Kurisawa, J. E. Chung, and Y. Y. Yang, "Synthesis and characterization of chitosan-g-poly(ethylene glycol)folate as a non-viral carrier for tumor-targeted gene delivery," Biomaterials, vol. 28, no. 3, pp. 540-549, 2007.

[32] C. P. Leamon and J. A. Reddy, "Folate-targeted chemotherapy," Advanced Drug Delivery Reviews, vol. 56, no. 8, pp. 1127-1141, 2004.

[33] D. Dubé, M. Francis, J. C. Leroux, and F. M. Winnik, "Preparation and tumor cell uptake of poly(N-isopropylacrylamide) folate conjugates," Bioconjugate Chemistry, vol. 13, no. 3, pp. 685-692, 2002.

[34] S. H. Kwon, S. Y. Kim, K. W. Ha et al., "Pharmaceutical evaluation of genistein-loaded pluronic micelles for oral delivery," Archives of Pharmacal Research, vol. 30, no. 9, pp. 1138-1143, 2007.

[35] Y. Ge, Y. Zhang, S. He, F. Nie, G. Teng, and N. Gu, "Fluorescence modified chitosan-coated magnetic nanoparticles for high-efficient cellular imaging," Nanoscale Research Letters, vol. 4, no. 4, pp. 287-295, 2009.
[36] T. M. Don, C. Y. Chuang, and W. Y. Chiu, "Studies on the degradation behavior of chitosan-g-poly (acrylic acid) copolymers," Tamkang Journal of Science and Engineering, vol. 5, no. 4, pp. 235-240, 2002.

[37] A. Grenha, B. Seijo, and C. Remuñán-López, "Microencapsulated chitosan nanoparticles for lung protein delivery," European Journal of Pharmaceutical Sciences, vol. 25, no. 4-5, pp. 427-437, 2005.

[38] J. Wu, Y. Lu, A. Lee et al., "Reversal of multidrug resistance by transferrin-conjugated liposomes co-encapsulating doxorubicin and verapamil," Journal of Pharmacy and Pharmaceutical Sciences, vol. 10, no. 3, pp. 350-357, 2007.

[39] A. V. Kabanov, P. Lemieux, S. Vinogradov, and V. Alakhov, "Pluronic block copolymers: novel functional molecules for gene therapy," Advanced Drug Delivery Reviews, vol. 54, no. 2, pp. 223-233, 2002.

[40] G. Acharya and K. Park, "Mechanisms of controlled drug release from drug-eluting stents," Advanced Drug Delivery Reviews, vol. 58, no. 3, pp. 387-401, 2006.

[41] C. Manaspon, K. Viravaidya-Pasuwat, and N. Pimpha, "Synthesis and characterization of folate-targeted chitosan coreshell nanoparticles for doxorubicin delivery," in Proceedings of the Biomedical Engineering International Conference (BMEiCON '09), pp. 200-204, 2009.

[42] P. E. Strømhaug, T. O. Berg, T. Gjøen, and P. O. Seglen, "Differences between fluid-phase endocytosis (pinocytosis) and receptor-mediated endocytosis in isolated rat hepatocytes," European Journal of Cell Biology, vol. 73, no. 1, pp. 28-39, 1997.

[43] J. Davda and V. Labhasetwar, "Characterization of nanoparticle uptake by endothelial cells," International Journal of Pharmaceutics, vol. 233, no. 1-2, pp. 51-59, 2002.

[44] E. R. Gillies and J. M. J. Fréchet, "pH-responsive copolymer assemblies for controlled release of doxorubicin," Bioconjugate Chemistry, vol. 16, no. 2, pp. 361-368, 2005.

[45] N. S. Melik-Nubarov and M. Y. Kozlov, "Evaluation of partition coefficients of low molecular weight solutes between water and micelles of block copolymer of ethylene oxide based on dialysis kinetics and fluorescence spectroscopy," Colloid and Polymer Science, vol. 276, no. 5, pp. 381-387, 1998.

[46] Y. Tian, L. Bromberg, S. N. Lin, T. Alan Hatton, and K. C. Tam, "Complexation and release of doxorubicin from its complexes with pluronic P85-b-poly(acrylic acid) block copolymers," Journal of Controlled Release, vol. 121, no. 3, pp. 137-145, 2007.

[47] E. Hecht and H. Hoffmann, "Interaction of ABA block copolymers with ionic surfactants in aqueous solution," Langmuir, vol. 10, no. 1, pp. 86-91, 1994.

[48] E. Hecht, K. Mortensen, M. Gradzielski, and H. Hoffmann, "Interaction of ABA block copolymers with ionic surfactants: influence on micellization and gelation," Journal of Physical Chemistry, vol. 99, no. 13, pp. 4866-4874, 1995.

[49] R. Barreiro-Iglesias, C. Alvarez-Lorenzo, and A. Concheiro, "Chitosan/sodium dodecylsulfate interactions calorimetric titration and consequences on the behaviour of solutions and hydrogel beads," Journal of Thermal Analysis and Calorimetry, vol. 82, pp. 499-505, 2005.

[50] A. Dedinaite and M. Ernstsson, "Chitosan-SDS interactions at a solid-liquid interface: effects of surfactant concentration and ionic strength," Journal of Physical Chemistry B, vol. 107, no. 32, pp. 8181-8188, 2003.

[51] R. A. A. Muzzarelli, "Human enzymatic activities related to the therapeutic administration of chitin derivatives," Cellular and Molecular Life Sciences, vol. 53, no. 2, pp. 131-140, 1997. 
[52] Z.-Q. Su, H.-L. Zhang, S.-H. Wu, Y. Tao, and L.-Q. Zang, "Preparation and characterization of water-soluble chitosan nanoparticles as protein delivery system," Journal of Nanomaterials, vol. 2010, Article ID 898910, 5 pages, 2010.

[53] A. Srinatha, J. Pandit, and S. Singh, "Ionic cross-linked chitosan beads for extended release of ciprofloxacin: in vitro characterization," Indian Journal of Pharmaceutical Sciences, vol. 70, no. 1, pp. 16-21, 2008.

[54] R. Savić, L. Luo, A. Eisenberg, and D. Maysinger, "Micellar nanocontainers distribute to defined cytoplasmic organelles," Science, vol. 300, no. 5619, pp. 615-618, 2003.

[55] O. Soga, C. F. Van Nostrum, M. Fens et al., "Thermosensitive and biodegradable polymeric micelles for paclitaxel delivery," Journal of Controlled Release, vol. 103, no. 2, pp. 341-353, 2005.

[56] H. L. Wong, A. M. Rauth, R. Bendayan et al., "A new polymerlipid hybrid nanoparticle system increases cytotoxicity of doxorubicin against multidrug-resistant human breast cancer cells," Pharmaceutical Research, vol. 23, no. 7, pp. 1574-1585, 2006. 

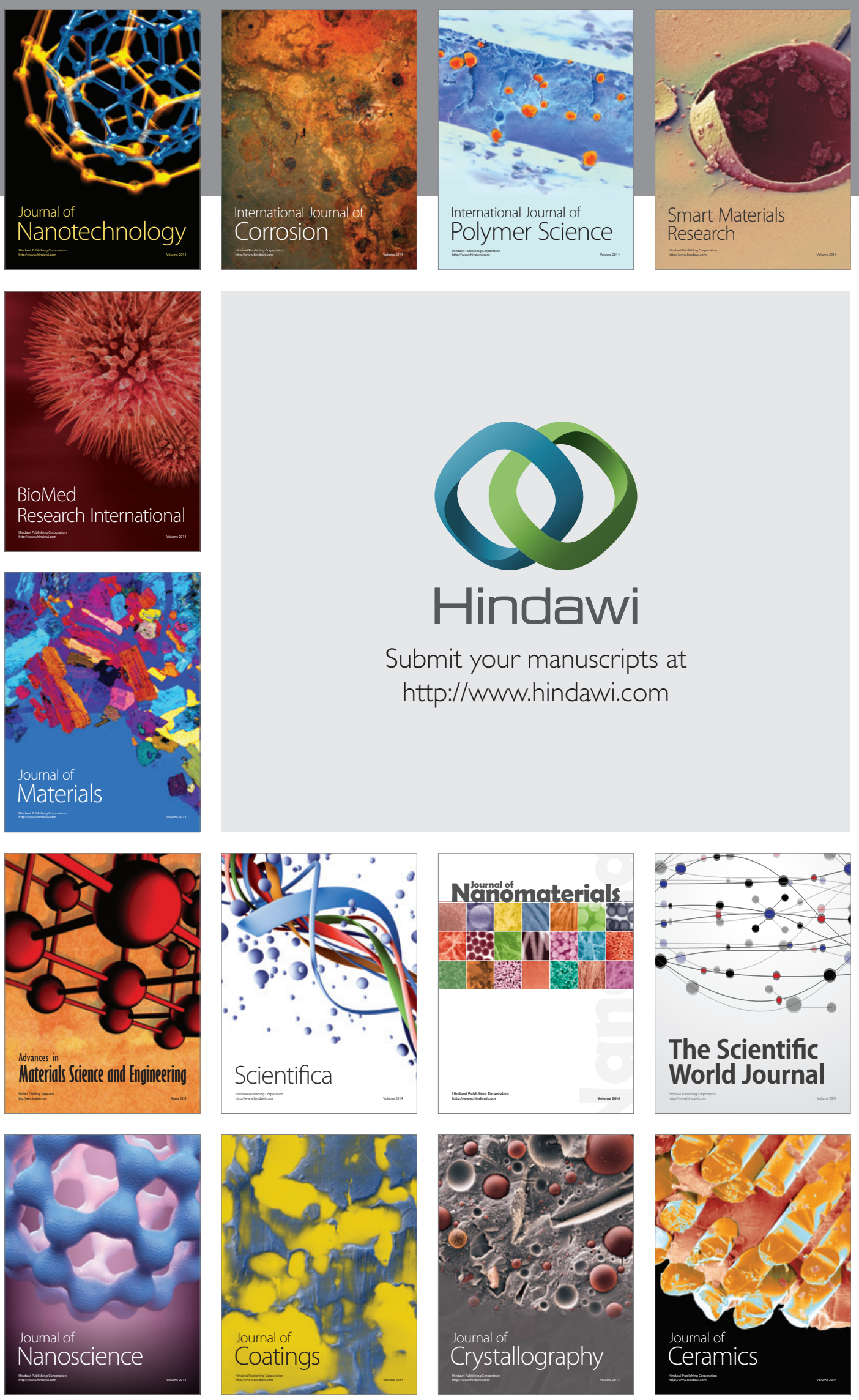

The Scientific World Journal

Submit your manuscripts at

http://www.hindawi.com

\section{World Journal}

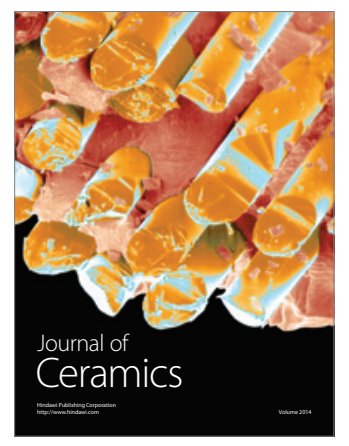

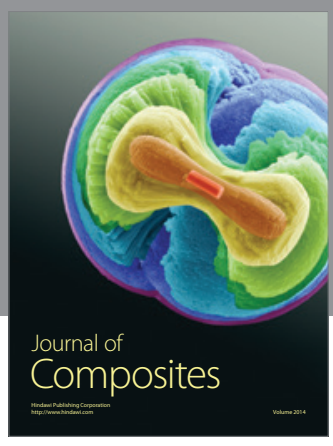
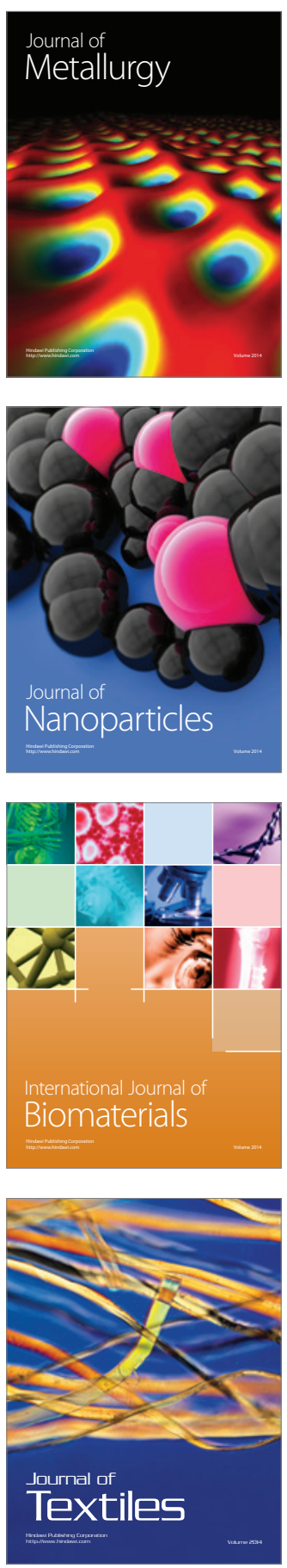